\title{
Estudios sobre macroinvertebrados acuáticos en América Latina: avances recientes y direcciones futuras
}

\author{
Alonso Ramírez ${ }^{1} \&$ Pablo E. Gutiérrez-Fonseca ${ }^{2}$ \\ 1. Departamento de Ciencias Ambientales, Universidad de Puerto Rico, P.O. Box 190341, San Juan, Puerto Rico 00919; \\ aramirez@ramirezlab.net \\ 2. Departamento de Biología, Universidad de Puerto Rico, San Juan, Puerto Rico 00919; gutifp@gmail.com
}

Recibido 12-XII-2013. Corregido 20-I-2014. Aceptado 13-II-2014.

\begin{abstract}
Studies on Latin American freshwater macroinvertebrates: recent advances and future directions. Latin America is an active scientific research area, in particular with respect to the study of freshwater macroinvertebrates. The present serves as an introduction to a special issue that highlights recent research projects on macroinvertebrates in Latin America. As part of this introduction, we conducted a literature analysis of the last 14 years of publications from the region that highlights the steady increase in publications on macroinvertebrates. Most studies from 2000-2013 were conducted in Brazil, Colombia, Argentina, and Costa Rica, and were focused on taxonomy and different issues related to biodiversity and distribution. There was a tendency for the use of local low-impact journals, but high impact publications were also found. This special issue contributes with 18 studies conducted in eight different countries. Two major topics are covered in the special issue, the ecology and natural history of aquatic macroinvertebrates and their use in the evaluation of anthropogenic impacts to aquatic ecosystems. Based on the literature review and contributions included in the issue, we discuss research needs for the region. Identified needs include: (1) to continue emphasizing taxonomic research, (2) assess mechanisms responsible for changes in biodiversity, (3) assess the role of macroinvertebrates in ecosystem processes and function, (4) improve biomonitoring efforts beyond unimetric indices, (5) the need for an ecosystem perspective, and (6) establishing long-term studies. This special issue is an initial effort to advance our knowledge on freshwater macroinvertebrates in Latin America. Rev. Biol. Trop. 62 (Suppl. 2): 9-20. Epub 2014 April 01.
\end{abstract}

Key words: aquatic insects, rivers, lakes, wetlands, state of knowledge, Latin America, macroinvertebrates.

Latinoamérica comprende la mayor parte del continente Americano, incluyendo una porción de las islas del mar Caribe. Latitudinalmente, la región forma un gradiente desde el norte de México hasta la región Patagónica, cubriendo una gran variedad de biomas que incluyen: bosques tropicales, selvas, zonas Andinas, sabanas, Sertao, praderas y desiertos. En cada bioma se encuentran una variedad de ecosistemas que difieren, entre otras cosas, en la precipitación que reciben; la cual no se distribuye uniformemente a través del gradiente latitudinal. La combinación del gradiente latitudinal y las diferencias en precipitación entre biomas, resulta en una alta diversidad de ecosistemas de agua dulce tales como ríos, lagos, humedales, e inclusive huecos en árboles con agua. Esta diversidad de ambientes y ecosistemas resulta en una alta diversidad de organismos acuáticos. Los macroinvertebrados (insectos, decápodos, etc.) son uno de los grupos más diversos dentro de los ambientes de agua dulce de América Latina. Muchos de los grupos de organismos acuáticos alcanzan su máxima diversidad dentro del gradiente latitudinal comprendido por esta región (Vinson \& Hawkins, 2003) y por ende han sido el foco de gran cantidad de estudios. A pesar de ello, los vacíos de información son evidentes, siendo uno de los principales obstáculos para el 
manejo y conservación de los recursos hídricos de América Latina.

La región siempre ha sido atractiva para los investigadores, en particular por la gran diversidad de ambientes y organismos que en ella se encuentran, por lo que América Latina es una zona de notoria actividad científica. Evidencia de esto se refleja en el número de proyectos especiales que se han publicado. Los últimos 25 años han generado varios esfuerzos para promover y divulgar la actividad científica en la región (particularmente en la región tropical). La revista Freshwater Sciences (antes Journal of the North American Benthological Society) ha publicado cuatro compendios de artículos. El primero fue editado por Stanford \& Covich (1988) y cubre temas sobre la estructura y función de las comunidades en ríos tropicales, incluyendo la formulación de teorías de distribución de los organismos. En el segundo compendio, Jackson \& Sweeney (1995) incluyeron artículos taxonómicos y de historia de vida de insectos neotropicales, así como investigaciones sobre el exporte de nutrientes y el efecto de los pesticidas en los insectos acuáticos. El tercer proyecto fue editado por Wantzen, Ramirez \& Winemiller (2006) y abarcó una gran variedad de temas, incluyendo variación espacio-temporal, procesamiento de detrito, descomposición de hojarasca, entre otros. Finalmente, el más reciente fue editado por Boyero, Ramírez, Dudgeon \& Pearson (2009) con un enfoque en trabajos de biogeoquímica y procesos ecológicos. Otros esfuerzos incluyen una selección de artículos publicados en Aquatic Conservation: marine and freshwater ecosystems. Bajo la edición de Moulton \& Wantzen (2006), el compendio incluyó trabajos enfocados principalmente al efecto de la generación de electricidad y otros disturbios antropogénicos sobre los patrones de diversidad y las especies migratorias. Finalmente, Contador, Kenndy \& Rozzi (2012) examinaron las tendencias de la actividad científica a nivel global y enfatizaron contrastes con Suramérica. Los autores encontraron que un alto porcentaje de los estudios realizados entre 1975 al 2010 corresponde a taxonomía y procesos ecológicos; donde los grupos más estudiados fueron Odonata y Diptera, con un importante incremento en Ephemeroptera, Plecoptera y Trichoptera.

A pesar del alto valor y atractivo de la región Latinoamericana para la investigación científica, existen serias limitaciones que obstaculizan una mayor productividad por parte de los científicos que trabajan en esta región. Entre los problemas más comunes tenemos la falta de acceso a recursos taxonómicos, incluyendo literatura y expertos en macroinvertebrados acuáticos. También tenemos el tema del acceso a la literatura en general, la cual es generalmente accesible solo por contacto directo con los autores de las publicaciones. También existe una interacción limitada entre los colegas de la región; dentro de áreas especializadas (e.g., taxonomía de Odonata) la comunicación es buena, mas es difícil para los no especialistas conseguir la información de contacto de los expertos. En respuesta a estas necesidades se han organizado recientemente varios proyectos. En febrero del 2012 se organizó en Costa Rica un congreso sobre macroinvertebrados de agua dulce en América Latina. Durante el congreso se discutieron las limitaciones más comunes que enfrentan los investigadores de la región. De las interacciones del congreso surgió el interés de crear una red que enlace a todos los interesados en estudiar los macroinvertebrados de agua dulce y sus ambientes. Posteriormente fue creada la red Macrolatinos@ (http://macrolatinos.ramirezlab.net/) con el propósito de facilitar la comunicación entre investigadores y también desarrollar proyectos de interés común.

El presente número especial es parte de estos proyectos dirigidos a resaltar la investigación en América Latina. Los manuscritos incluidos no se limitan a las presentaciones en el congreso en Costa Rica, pero se cubren muchos de los temas allí presentados. Paralelo al presente, también se editó un número especial en la revista Dugesiana cubriendo temas similares (Dominguez \& Springer, 2013). En esta introducción al número especial hacemos énfasis en tres temas. Primero, presentamos un 
análisis de la literatura sobre macroinvertebrados acuáticos en América Latina publicada en los últimos 14 años (2000-2013). El análisis se hace con el fin de identificar patrones dentro de las publicaciones en cuanto al tema de investigación y los autores de los trabajos. El objetivo es identificar áreas que necesitan atención y poner el presente número especial en un contexto actual. Segundo, hacemos una breve introducción al número especial, sus contribuciones y los vacíos de información que contribuyen a llenar. Tercero, basados en el análisis de publicaciones y en las contribuciones del número especial, discutimos áreas de investigación que necesitan mayor atención y sugerimos algunas direcciones futuras que permitirían avanzar la investigación de los macroinvertebrados acuáticos en América Latina.

\section{AVANCES RECIENTES}

Con el propósito de determinar patrones en los temas de investigación que se han publicado en los últimos años, se realizó una recopilación de literatura que cubre las publicaciones sobre macroinvertebrados acuáticos en Latinoamérica durante los últimos 14 años. Se utilizaron las siguientes palabras claves en el buscador de Google Scholar: biomonitoreo acuático, ecología acuática, macroinvertebrados acuáticos, taxonomía, e inventarios faunísticos. También se utilizó el nombre de cada uno de los órdenes de insectos acuáticos y se hizo la búsqueda en español e inglés. Adicionalmente a la búsqueda en inglés se agregaron las siguientes palabras claves: "tropical streams" y "freshwater ecology". De los resultados obtenidos se seleccionaron aquellos cuya investigación fue realizada en América Latina. Los artículos recopilados se evaluaron y clasificaron para determinar tendencias en cuanto a contenido para determinar los temas más comunes, los grupos taxonómicos y los ambientes mejor estudiados. Además, se evaluó la información de los investigadores y la revista utilizada para determinar los países que más han aportado al conocimiento actual, el idioma más utilizado, y el tipo de revista seleccionado.
Un total de 500 artículos fueron recopilados, publicados desde el 2000 al 2013. Este número es conservador, ya que el buscador de Google probablemente no incluye todas las revistas científicas de la región o bien las palabras usadas no cubren todos los posibles trabajos. A pesar de ello, los trabajos incluyeron una alta diversidad de publicaciones, algunas en revistas de interés local (e.g., revistas de museos o de universidades) hasta revistas reconocidas internacionalmente (e.g., Annual Review of Entomology). En cuanto a la cantidad de publicaciones por año, se observó un claro aumento en la cantidad de publicaciones desde el inicio de la década. El 2000 tuvo la menor proporción de publicaciones, pero se observó un aumento lineal hasta un máximo en el año 2010 (Fig. 1). El 2006 y el 2008 fueron dos años altamente productivos y similares al 2010. La proporción de trabajos disminuyó un poco en el 2012, pero volvió a remontar para el 2013 (Fig. 1). Este patrón puede ser una mezcla de aumento en la productividad científica y los esfuerzos que las revistas hacen por hacer sus trabajos disponibles en línea.

La mayoría de las investigaciones tuvieron como sitio de estudio alguna localidad en Brasil (35.0\%). En segundo lugar fueron localidades en Colombia en un $13.7 \%$ de los artículos, seguidos por localidades en Argentina y Costa Rica en un 9.7 y $6.8 \%$, respectivamente (Fig. 2A). El 35\% restante de los trabajos fueron realizados en diferentes localidades de 18 países. Este patrón es interesante ya que el $65 \%$ de las investigaciones se realizaron en tres países de amplia extensión territorial y uno de los más pequeños. En cuanto al enfoque de los trabajos, se encontró que la taxonomía continua siendo uno de los temas más importantes en la región (28.1\%), seguidos por estudios cuyo objetivo fue cuantificar la biodiversidad de la región, ya sea evaluando patrones de diversidad o bien con inventarios faunísticos $(22.7 \%$, Fig. 2B). Un $11.1 \%$ estuvo enfocado en patrones de distribución de grupos acuáticos en particular. El tema de biomonitoreo, que ha recibido mucha atención en años recientes, comprendió el $10.3 \%$ de las publicaciones, un porcentaje 


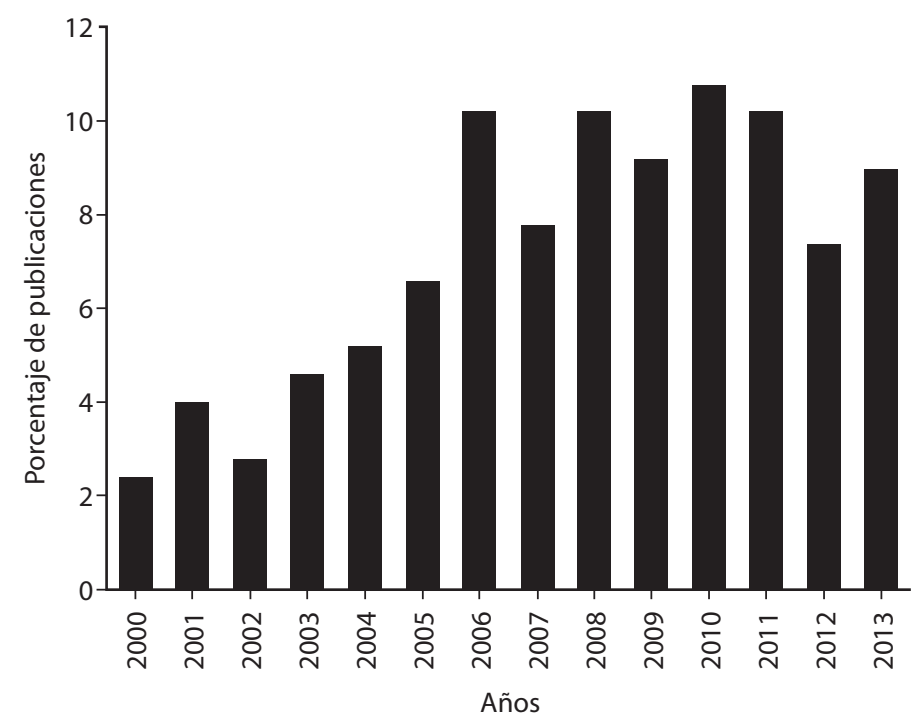

Fig. 1. Porcentaje de publicaciones por año $(n=500)$ en el periodo 2000-2013 que se enfocaron en macroinvertebrados acuáticos en América Latina. La revisión se hizo con el buscador de Google Scholar y las palabras clave: biomonitoreo acuático, ecología acuática, macroinvertebrados acuáticos, taxonomía, inventarios faunísticos, "tropical streams", y "freshwater ecology".

Fig. 1. Percent of publications by year $(n=500)$ from 2000-2013 focused on aquatic macroinvertebrates in Latin America. The literature review was conducted using Google Scholar and the keywords (in Spanish): biomonitoreo acuático, ecología acuática, macroinvertebrados acuáticos, taxonomía, inventarios faunísticos, "tropical streams", and "freshwater ecology”.

que probablemente aumente en el futuro. El restante $27.8 \%$ se enfocó en diferentes temas, como la ecología e historia natural (Fig. 2B). En cuanto a los órdenes de insectos acuáticos más estudiados, Ephemeroptera recibió más atención con un $24.9 \%$ de los artículos, mientras que Trichoptera fue el segundo lugar con un $15.3 \%$ (Fig. 2C). Este patrón es similar al reportado para Suramérica por Contador et al. (2012). Finalmente, la mayoría de los trabajos fueron realizados en ambientes lóticos (e.g., arroyos o ríos), con el resto de los ambientes acuáticos recibiendo una cantidad mínima de atención (Fig. 2D).

En cuanto al idioma utilizado, la mayoría de los trabajos siguieron el patrón general de las ciencias y fueron publicados en inglés (53.6\%), seguidos por español (35.8\%) y finalmente portugués $(11.0 \%$, Fig. 3A). La mayor parte de los autores tuvieron una afiliación (e.g., dirección institucional) basada en Brasil (Fig. 3B). Seguido por afiliaciones en Argentina, Colombia y Estados Unidos. Este patrón indica la ubicación geográfica de los autores, y no es tan diferente al orden basado en los sitios de estudio (Fig. 2A). Sin embargo, Costa Rica pasa a ser el $7^{\circ}$ lugar en lugar del $4^{\circ}$ y Estados Unidos aparece dentro de los cinco primeros países (Fig. 3B). La cantidad de co-autores por publicación ofreció una idea sobre el grado de colaboración en la realización de las publicaciones. La mayor parte de las publicaciones siguió el patrón tradicional de uno o dos autores por trabajo (47.7\%). Sin embargo, es interesante ver como la cantidad de trabajos con 3-5 autores comprendió una cantidad similar, con un $46.1 \%$, reflejando quizás un aumento en la complejidad de los temas. Finalmente, trabajos de más de cinco autores reflejan colaboraciones regionales o internacionales, o bien proyectos complejos. Solo un $6.2 \%$ de los trabajos fueron realizados por grupos de más de 5 autores. Para evaluar el tipo de revista utilizado en las publicaciones se utilizó el factor de impacto como indicador. El 19.4\% de las publicaciones apareció en revistas no indexadas (Fig. 3C). La 

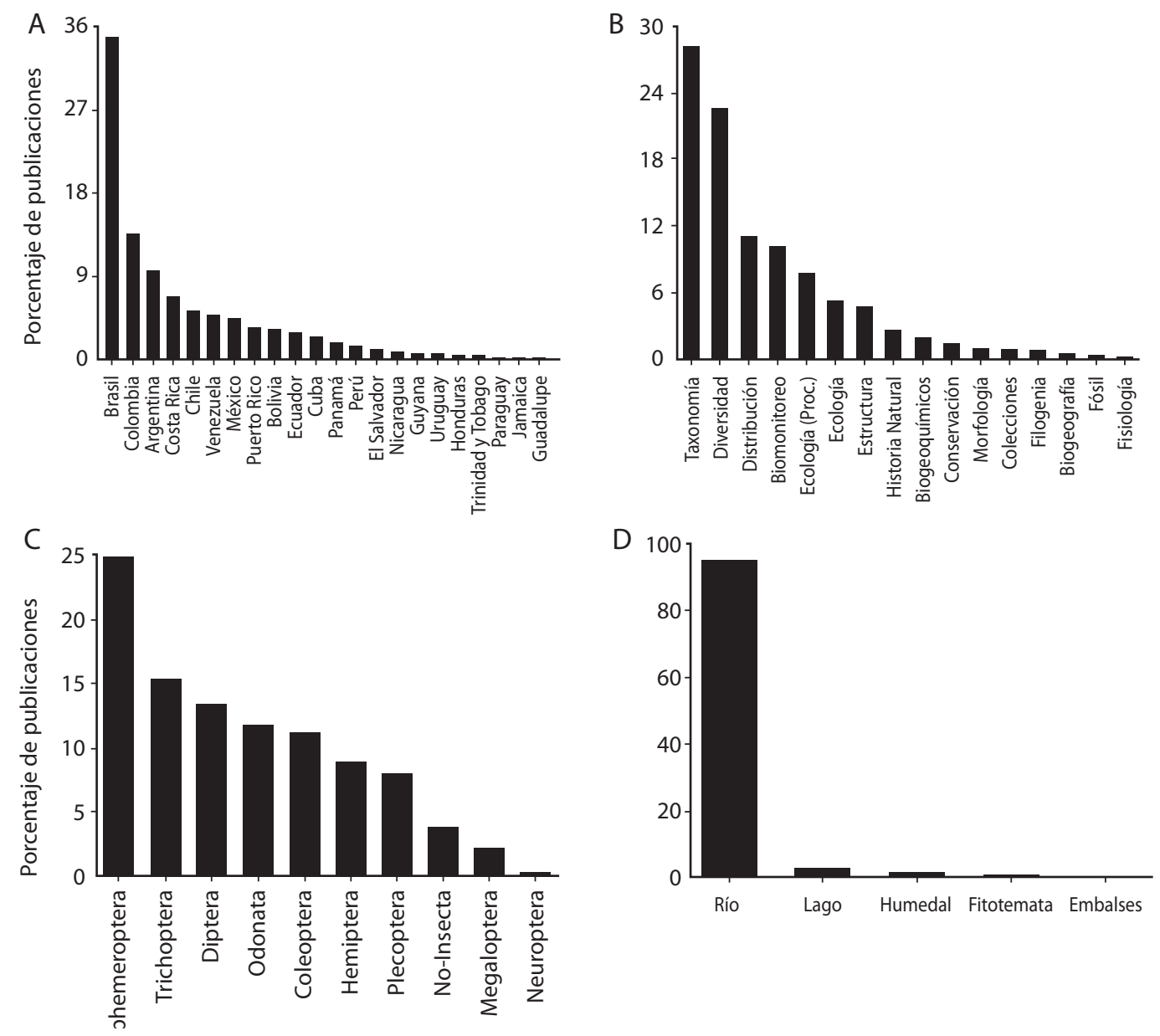

Fig. 2. Resumen del enfoque de los artículos encontrados en la revisión de literatura. Las barras representan el porcentaje por categoría. A- Países donde fueron realizados los estudios. B- Temas estudiados. Ecología (Proc) se refiere a procesos ecológicos. C- Ordenes de macroinvertebrados estudiados. D- Ambientes estudiados.

Fig. 2. Summary of the topics studied in the publications found in the literature review. Bars are percentages per category. A- Countries where the research was conducted. B- Study topics. Ecología (Proc) refers to ecological processes. CMacroinvertebrate orders studied. D- Aquatic environments studied.

mayor parte $(64.3 \%)$ fue publicada en revistas con un factor de impacto menor a uno. Un $16.2 \%$ en revistas de "mediano" o "alto impacto", con factores de más de uno (Fig. 3C). También calculamos el factor de impacto promedio para cada país, usando la afiliación del primer autor y solo incluyendo países con tres o más publicaciones durante el periodo de estudio. El patrón resultante fue diferente a los anteriores. Entre los cinco primeros países con factor de impacto promedio alto, solo aparece un país latinoamericano (Fig. 3D). Lo cual indica que la mayoría de las publicaciones producidas por autores con afiliación en América Latina aparecieron en revistas con factor de impacto menor a uno. La predominancia de publicaciones en revistas de mediano y bajo impacto puede tener varias razones. Por un lado, nuestra búsqueda resultó en un alto número de trabajos taxonómicos, los que generalmente se publican en revistas con factores de impacto menores a uno (v.g., Zootaxa tiene un factor de impacto de 0.97). Además, se observa una tendencia a publicar en revistas regionales o locales, las 

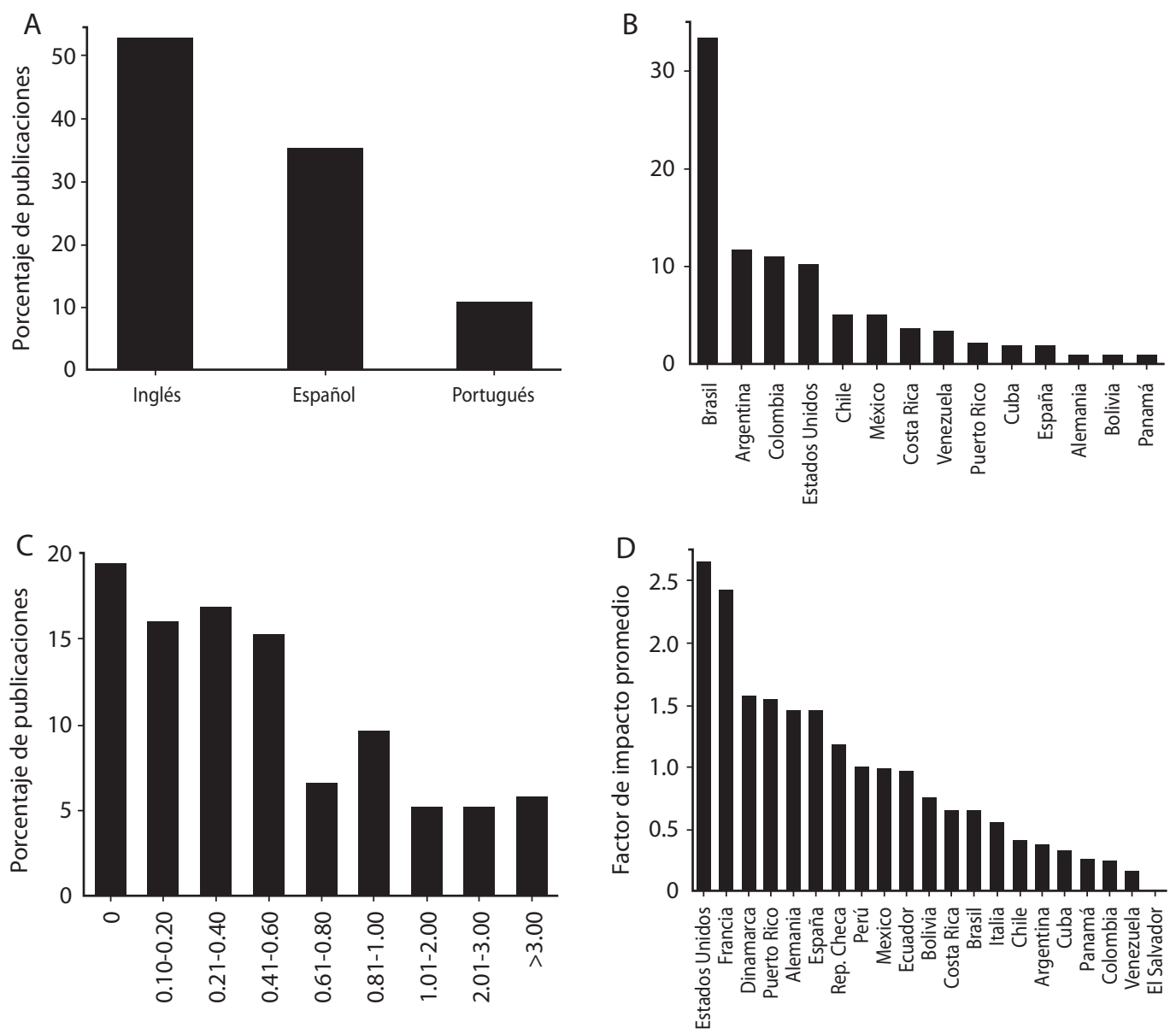

Fig. 3. Resumen de la información sobre los artículos encontrados en la revisión de literatura. Las barras representan el porcentaje por categoría. A- Idioma de la publicación. B- País de origen del primer autor, basado en la dirección de contacto. C- Porcentaje de trabajos por categoría de factor de impacto de la revista. D- Factor de impacto promedio por país, para aquellos países con más de tres publicaciones.

Fig. 3. Summary of the topics studied in the publications found in the literature review. Bars are percentages per category. A- Language used in the publication. B- Country of affiliation of the first author, based on the contact address. C- Percent of studies by categories of journal impact factor. D- Mean impact factor by country, only those with more than three publications were included.

que tienen factores de impacto bajos. Finalmente, una proporción alta de los estudios conducidos por extranjeros son ecológicos, cuyas revistas tienden a tener factores de impacto medios o altos. En general, esto no significa que en los países latinos no se publiquen trabajos de alto impacto, simplemente indica las tendencias en la región.

\section{EL NÚMERO ESPECIAL}

El presente número especial refleja la diversidad de investigaciones que se realizan en la actualidad en diferentes regiones de América Latina. El número incluye 18 trabajos que presentan información sobre macroinvertebrados acuáticos recolectada en ocho países 
(Colombia, Costa Rica, Ecuador, Guatemala, México, Perú, Puerto Rico y Venezuela). Los trabajos se pueden dividir en dos grupos con similar número de publicaciones, que de cierta forma reflejan la realidad de la región. Un grupo de trabajos se enfoca en la ecología y la historia natural de los macroinvertebrados acuáticos, haciendo patente necesidad de entender aspectos básicos de este grupo de organismos y la función que juegan en los ecosistemas acuáticos. El segundo grupo se enfoca en temas asociados a evaluar impactos antropogénicos sobre los ecosistemas acuáticos. Dentro de este grupo sobresale particularmente el uso de índices de evaluación biótica y los programas de biomonitoreo.

Los trabajos sobre historia natural y ecología contienen estudios sobre la autoecología de los organismos, como por ejemplo análisis de los hábitos alimentarios (Guzmán-Soto \& Tamarís-Turizo, 2014), el efecto de los factores ambientales sobre la selección de hábitat (Vásquez-Ramos, Guevara-Cardona, ReinosoFlórez, 2014), y sobre cómo la presencia de macroconsumidores (v.g., camarones) influencia las estrategias de vida de los insectos (Macías, Colón-Gaud, Duggins \& Ramírez, 2014). El trabajo de Blanco, Tamayo \& Scatena (2014) resalta la variabilidad morfológica que caracteriza ciertos grupos de macroinvertebrados y su importancia en los estudios acuáticos. Otro grupo de trabajos se enfoca en el efecto de los factores ambientales sobre la diversidad de macroinvertebrados. Estos trabajos se caracterizaron por estudiar diferentes ambientes, como ríos (Torres-García, Pérez-Valladares, HerreríaDiego \& Pineda-López, 2014), lagos (RicoSánchez, Rodríguez-Romero, López-López \& Sedeño-Díaz, 2014) y zonas de páramo en las montañas de Colombia (Latorre-Beltrán, Novelo-Gutiérrez \& Favila, 2014).

Dentro de los trabajos sobre ecología, tres estudios hacen énfasis en entender la importancia de los macroinvertebrados en procesos ecológicos, como la descomposición de la hojarasca que entra de la zona ribereña. El efecto que tienen diferentes usos de terreno sobre los macroinvertebrados que colonizan la hojarasca y sobre el proceso de descomposición como tal fue estudiado en bosques nubosos en México (Astudillo, Ramírez, Novelo-Gutiérrez \& Vázquez 2014) y en un gradiente de bosques a zonas urbanas en Puerto Rico (Torres \& Ramírez, 2014). Ambos estudios encontraron importantes efectos de los cambios en uso de terreno sobre el procesamiento de la hojarasca en los ríos. Por otro lado, los bosques tropicales tienen una alta diversidad de especies de plantas ribereñas, y diversos tipos de organismos acuáticos que las consumen. En este contexto, Rincón \& Covich (2014) estudiaron la función de diferentes grupos de macroinvertebrados (insectos vs. camarones) en el procesamiento de diferentes especies de hojas.

Finalmente, los macroinvertebrados acuáticos no solo consumen ciertos tipos de recursos, si no que los consumen de manera particular. La forma en que estos organismos obtienen su alimento nos ayuda a determinar o deducir la función que éstos tienen en los ecosistemas acuáticos. Las características y la forma en que se clasifican los grupos funcionales alimentarios se discute en Ramírez \& Gutiérrez-Fonseca (2014). El uso de los grupos funcionales es una herramienta que ayuda a determinar la diversidad funcional de los macroinvertebrados. En contraste, cuando el objetivo es entender el tipo de recursos consumido o la base alimentaria (e.g., Guzmán-Soto \& Tamarís-Turizo, 2014), se recomienda usar la clasificación de gremios tróficos, que hace énfasis en el tipo de alimento y no en la forma en que se obtiene.

En el segundo grupo de trabajos, enfocados en los impactos antropogénicos sobre los ecosistemas acuáticos y los macroinvertebrados, encontramos dos contribuciones que hacen énfasis en impactos comunes en la región, las represas y la actividad agropecuaria. La construcción de represas para la producción hidroeléctrica es una actividad que continúa en aumento en América Latina (Ramírez, Pringle \& Wantzen, 2008). Los impactos en los ecosistemas lóticos y en los ensamblajes de macroinvertebrados son diversos y algunas veces no tan fuertes como se esperaría (Chaves-Ulloa, 
Umaña-Villalobos \& Springer, 2014). Otra actividad con importantes impactos sobre los ecosistemas acuáticos es la agricultura. Los impactos de esta actividad son complejos, ya que incluyen cambios en la fisicoquímica del agua, la disponibilidad de hábitat y en los ensamblajes de macroinvertebrados (Giraldo, Chará, Zúñiga, Chará-Serna \& Pedraza, 2014).

La mayoría de los trabajos dentro del segundo grupo de publicaciones se orienta en el uso de índices de evaluación de la calidad del agua o la integridad biótica de los ríos. Piñón Flores, Pérez Munguía, Torres García \& Pineda López (2014) hacen énfasis en el uso de diversas variables fisicoquímicas y el ensamblaje de macroinvertebrados como herramientas para determinar la integridad biótica de los ríos de la subcuenca del Río Chiquito en México. Por otro lado, dos trabajos hacen énfasis en el desarrollo o mejora de índices bióticos. Forero, Longo, Ramírez \& Chalar (2014) presentan un nuevo índice para la cuenca del Río Negro, Colombia, mientras que Rios-Touma, Acosta \& Prat (2014) revisan los valores de tolerancia utilizados en las regiones andinas y en el índice biótico para la región (Andean Biotic Index). Finalmente, dos estudios nos recuerdan la importancia de entender las limitaciones de los índices en cuanto al esfuerzo de muestreo, ambos enfocados a evaluar diferentes versiones del índice BMWP. Gutiérrez-Fonseca \& Lorion (2014) compararon la respuesta del índice BMWP-CR utilizando un muestreador Surber y un colador de cocina. Los autores encontraron que el puntaje del índice difirió de acuerdo al método empleado. Por otro lado, Reyes-Morales \& Springer (2014) evaluaron 12 ríos en la cuenca del Lago Atitlán con tres diferentes tiempos de recolecta. Las autoras encontraron diferencias importantes en las evaluaciones obtenidas según los tiempos de recolecta.

\section{DIRECCIONES FUTURAS}

A pesar de la creciente cantidad de publicaciones desde el 2000, es claro que algunas áreas de investigación han recibido más atención que otras y algunas faltan todavía por explorarse.
Basados en nuestra revisión de literatura, proponemos las siguientes áreas como aquellas que necesitan más atención en estudios futuros:

Taxonomía y Sistemática: A pesar de que la taxonomía sigue recibiendo profusa atención en América Latina, creemos que la misma debe seguir siendo una de las prioridades de trabajo. En especial en lo que respecta a mejorar la taxonomía de los estados inmaduros y su asociación con los adultos, los que en general son utilizados en estudios ecológicos y de bioindicación. Además, algunos grupos siguen recibiendo poca atención y por ende continúan poco estudiados (v.g., los Blattaria semiacuáticos, algunas familias de Diptera). También hace falta más énfasis en la utilización de técnicas avanzadas de apoyo a los taxónomos, como las de código de barras, para ayudar a resolver la taxonomía de algunos grupos y de este modo resolver las relaciones filogenéticas entre grupos. Dentro de la taxonomía, también es necesario el desarrollo de herramientas de identificación (v.g., claves taxonómicas, técnicas particulares, cursos y talleres) que nos ayuden a aprender y también a entrenar nuevos taxónomos.

Diversidad de macroinvertebrados: $\mathrm{La}$ literatura incluye una cantidad apreciable de estudios sobre la diversidad taxonómica de los macroinvertebrados acuáticos. Sin embargo, pocos estudios hacen esfuerzos por entender los mecanismos que controlan o determinan estos patrones (v.g., factores ambientales, fisicoquímica de los ambientes). Muchos de los estudios se limitan a describir los patrones. Además, hace falta más énfasis en otros tipos de diversidad, a parte de la taxonómica, como por ejemplo diversidad funcional y diversidad filogenética. Un área de interés entonces sería el desarrollo de explicaciones mecanísticas sobre los factores que mantienen los diferentes tipos de biodiversidad en los ecosistemas dulceacuícolas de América Latina.

Ecología y procesos ecológicos: Muy pocos de los trabajos publicados y de los que 
se incluyen en este número especial se enfocan en temas ecológicos, relativo a la diversidad de temas disponibles para estudiar. Aspectos básicos de la autoecología de los organismos, como el tipo de alimento que consumen, la forma en que adquieren el alimento (grupo funcional), o bien la duración de los estadios larvales o de adulto son temas básicos que no conocemos de la mayoría de las especies de macroinvertebrados. Esta información es vital para entender la función de los macroinvertebrados dentro de sus ecosistemas y poder utilizarlos en otros tipos de estudios. Aspectos más avanzados, como la productividad secundaria o bien la función que tienen los macroinvertebrados en los procesos ecológicos son poco estudiados. La excepción es quizás el tema de la descomposición de la hojarasca, uno de los procesos ecológicos más estudiados actualmente. Sin embargo, pocos estudios tienen diseños que nos permitan saber el impacto de los macroinvertebrados en estos procesos. Entender la función de los macroinvertebrados dentro de sus ecosistemas debe ser una de nuestras prioridades futuras.

Biomonitoreo o bioindicación: La mayoría de los trabajos publicados sobre la evaluación de los ecosistemas acuáticos usando macroinvertebrados se basa en el uso de índices unimétricos. El índice BMWP en sus diferentes versiones o adaptaciones es quizás el más utilizado. El uso de estos índices ha sido sumamente importante en cuanto a resaltar la importancia de las evaluaciones con macroinvertebrados y en la educación ambiental. Sin embargo, hace falta moverse más allá de los índices unimétricos y buscar nuevas alternativas para evaluar los ecosistemas acuáticos. En especial, es importante buscar opciones que permitan evaluaciones sólidas, objetivas, e integrales (v.g., no limitadas a pocas variables). El uso de índices multimétricos puede ser una alternativa, aunque la limitada cantidad de información ecológica y de tolerancia a los factores ambientales que tenemos hace difícil desarrollar estos índices. Un tipo de metodología que amerita incorporarse a esta área de investigación es el uso de técnicas multivariadas. Las técnicas multivariadas se basan en conceptos ecológicos (e.g., nichos ecológicos), el uso de ecosistemas de referencia y la medición de variables ambientales (e.g., substratos, fisicoquímica; Bonada, Prat, Resh \& Statzner, 2006). Estos índices no requieren información del ciclo de vida, de la tolerancia a la contaminación o de la ecología de los macroinvertebrados. Por ende, son ideales para ser aplicados en áreas donde tenemos información limitada sobre los macroinvertebrados.

Visión de ecosistema: El utilizar una visión de cuenca está bien establecido dentro de los estudios acuáticos. Sabemos que la cobertura del terreno en la cuenca de un cuerpo de agua juega un papel fundamental determinando sus características. Sin embargo, todavía debemos hacer un mejor esfuerzo para enfocar nuestras investigaciones con macroinvertebrados en el contexto de sus ecosistemas. Muchos estudios se enfocan en los macroinvertebrados en aislamiento de otros componentes del ecosistema. Por ejemplo, la presencia de recursos (v.g., algas, detrito) y depredadores (v.g., peces, camarones) generalmente no son incorporados dentro de las investigaciones sobre la dinámica de los macroinvertebrados. Igualmente, relativamente pocos trabajos hacen mediciones que incorporen la variabilidad espacial y temporal que ocurre de forma natural en los ecosistemas. Entender la posición de los macroinvertebrados dentro de la dinámica de sus ecosistemas es prioritario para poder interpretar correctamente nuestros estudios.

Investigaciones a largo plazo: Los estudios a largo plazo (v.g., más de cinco años) son sumamente valiosos para entender cómo los fenómenos a grandes escalas (v.g., El NiñaLa Niña, cambios climático) afectan los ecosistemas acuáticos y los macroinvertebrados. Lamentablemente, son muy pocos los trabajos de este tipo que se encuentran en la literatura. Establecer y mantener un esfuerzo a largo plazo puede ser oneroso. Sin embargo, estudios simples pero consistentes en cuanto a métodos y esfuerzo de muestreo pueden generar datos 
valiosos (e.g., patrones de abundancia, diversidad a largo plazo), en especial si se les asocian variables ambientales (e.g., precipitación, temperatura, $\mathrm{pH}$ ). Este tipo de labor es la única forma de encontrar tendencias y patrones que serían imposibles de dilucidar con estudios a corto plazo.

\section{AGRADECIMIENTOS DEL NÚMERO ESPECIAL}

Reconocemos que existe una cantidad de limitaciones que obstaculizan el desarrollo de los estudios con macroinvertebrados acuáticos en nuestros países Latinoamericanos. Sin embargo, el presente número evidencia los esfuerzos que se realizan y la calidad de los trabajos. Creemos que es una indicación de la dirección futura y del aumento en la productividad por venir.

Este proyecto fue posible gracias a la participación y apoyo de muchas personas. Los autores que respaldaron el número con sus manuscritos y se afanaron en mejorar sus trabajos atendiendo los comentarios recibidos. Una cantidad todavía mayor de colaboradores que apoyaron el proyecto como revisores de manuscritos. La lista se presenta dentro de este número con un gran agradecimiento por su entusiasmo. Monika Springer y Eduardo Domínguez, editores del número en Dugesiana, gentilmente manejaron la edición del manuscrito sobre grupos funcionales, del cual somos autores. También le agradecemos a Ana Marcela Chará-Serna el procesamiento de los manuscritos y la correspondencia asociada con los números especiales de Macrolatinos@. A todos ellos, mil gracias.

La publicación de este número especial fue posible gracias a la Society for Freshwater Sciences (SFS). Agradecemos a su presidente Randy Fuller y a los directivos por reconocer el esfuerzo involucrado en el proyecto y por su apoyo al mismo. También agradecemos al Center for Applied Tropical Ecology and Conservation (CATEC) de la Universidad de Puerto Rico, recinto de Río Piedras, por patrocinar esta publicación.

\section{AGRADECIMIENTOS}

Nuestro sincero agradecimiento a Bethany Vázquez por su ayuda en el resumen y análisis de la información de los artículos científicos evaluados. El manuscrito se benefició de los comentarios proveídos por Rodolfo Novelo Gutiérrez y Eduardo Domínguez.

\section{RESUMEN}

América Latina es una región bastante activa en cuanto a la investigación científica, en particular con respecto al estudio de macroinvertebrados de agua dulce. La presente es una introducción al número especial que incluye proyectos recientes en América Latina. Como parte de esta introducción, realizamos un análisis de la literatura publicada en los últimos 14 años, la cual tiene un aumento continuo en las publicaciones sobre macroinvertebrados. La mayoría de los estudios en el periodo 2000-2013 fueron realizados en Brasil, Colombia, Argentina, y Costa Rica; y se enfocaron en la taxonomía y temas relacionados con la biodiversidad y la distribución. Se observó una tendencia a publicar en revistas de bajo impacto, pero también se encontraron publicaciones de alto impacto. El número especial incluye 18 trabajos de ocho países. Los temas principales fueron ecología e historia natural de los macroinvertebrados acuáticos y su uso en la evaluación de impactos antropogénicos sobre los ecosistemas acuáticos. Usando la revisión y las publicaciones de este suplemento discutimos algunas necesidades de investigación para la región, principalmente: (1) continuar con los estudios taxonómicos, (2) determinar los mecanismos responsables por los cambios en biodiversidad, (3) evaluar el papel de los macroinvertebrados en los procesos ecológicos, (4) llevar los esfuerzos de biomonitoreo más allá de los métodos unimétricos, (5) implementar una visión de ecosistema, y (6) establecer estudios a largo plazo. Este suplemento representa un esfuerzo inicial para mejorar nuestro conocimiento sobre los macroinvertebrados de agua dulce de América Latina.

Palabras clave: insectos acuáticos, ríos, lagos, humedales, estado del conocimiento, Latinoamérica, macroinvertebrados.

\section{REFERENCIAS}

Astudillo, M. R., Ramírez, A., Novelo-Gutiérrez, R., \& Vázquez, G. (2014). Descomposición de hojarasca en seis arroyos de Bosque Mesófilo de Montaña en la cuenca alta del río La Antigua, Veracruz, México. Revista de Biología Tropical, Vol. 62 (Supl. 2): 109-125. 
Blanco, J. F., Tamayo, S., \& Scatena. N. F. (2014). Variación fenotípica de la concha en Neritinidae (Gastropoda: Neritimorpha) en ríos de Puerto Rico. Revista de Biología Tropical, Vol. 62 (Supl. 2): 51-66.

Bonada, N., Prat, N., Resh, V. H., \& Statzner, B. (2006). Developments in aquatic insect biomonitoring: A comparative analysis of recent approaches. Annual Review Entomology, 51, 495-523.

Boyero, L., Ramírez, A., Dudgeon, D., \& Pearson, R. G. (2009). Are tropical streams really different? Journal of the North American Benthological Society, 28(2), 397-403.

Chaves-Ulloa, R., Umaña-Villalobos, G., \& Springer, M. (2014). Downstream effects of hydropower production on aquatic macroinvertebrate assemblages in two rivers in Costa Rica. Revista de Biología Tropical, Vol. 62 (Suppl. 2): 177-199.

Contador, T. A., Kenndy, J. H., \& Rozzi, R. (2012). The conservation status of southern South American

aquatic insects in the literatura. Biodiviersity and Conservation, 21(8), 2095-2107.

Dominguez, E., \& Springer, M. (2013). Macroinvertebrados Acuáticos de Latino América: Presentación. Dugesiana, 20: 209.

Forero, L. C., Longo, M., Ramírez, R. J. J., \& Chalar, G. (2014). Índice de calidad ecológica con base en macroinvertebrados acuáticos para la cuenca del río Negro (ICERN-MAE), Colombia. Revista de Biología Tropical, Vol. 62 (Supl. 2): 231-245.

Giraldo, L. P., Chará, J., Zúñiga, M. C., Chará-Serna, A. M., \& Pedraza, G. (2014). Impacto del uso del suelo agropecuario sobre macroinvertebrados acuáticos en pequeñas quebradas de la cuenca del río La Vieja (Valle del Cauca, Colombia). Revista de Biología Tropical, Vol. 62 (Supl. 2): 201-217.

Gutiérrez-Fonseca, P. E., \& Christopher M. L. (2014). Application of the BMWP-Costa Rica biotic index in aquatic biomonitoring: sensitivity to collection method and sampling intensity. Revista de Biología Tropical, Vol. 62 (Suppl. 2): 273-287.

Guzmán-Soto, C. J., \& Tamarís-Turizo, C. E. (2014). Hábitos alimentarios de organismos inmaduros de Ephemeroptera, Plecoptera y Trichoptera de la parte media de un río tropical de montaña. Revista de Biología Tropical, Vol. 62 (Supl. 2): 167-176.

Jackson, J. K., \& Sweeney, B. W. (1995). Research in tropical streams and rivers: introduction to a series of papers. Journal of the North American Benthological Society, 14, 2-4.

Latorre-Beltrán, I. T., Novelo-Gutiérrez, R., \& Favila. M. E. (2014). Diversidad genérica de Trichoptera (Insecta) en dos microcuencas del Páramo Rabanal Cundinamarca- Boyacá, Colombia). Revista de Biología Tropical, Vol. 62 (Supl. 2): 95-108
Macías, N. A., Colón-Gaud, C., Duggins, J. W., \& Ramírez. A. (2014). Do omnivorous shrimp influence mayfly nymph life history traits in a tropical island stream?. Revista de Biología Tropical, Vol. 62 (Supl. 2): $39-49$.

Moulton, T. P., \& Wantzen, K. M. (2006) Conservation of tropical streams special questions or conventional paradigms?. Aquatic Conservation: Marine and freshwater ecosystems, 16, 659-663.

Piñón Flores, M. A., Pérez Munguía, R. M., Torres García, U., \& Pineda López, R. (2014). Integridad biótica de la microcuenca del Río Chiquito, Morelia Michoacán, México, basada en el ensamblaje de macroinvertebrados acuáticos. Revista de Biología Tropical, Vol. 62 (Supl. 2): 219-229.

Ramírez, A., Pringle, C. M., \& Wantzen, K. M. (2008). Tropical river conservation. In D. Dudgeon (Ed.), Tropical Stream Ecology (pp. 285-304). London, UK: Elsevier Science.

Ramírez, A., \& Gutiérrez-Fonseca, P. E. (2014). Functional feeding groups of aquatic insect families in Latin America: a critical analysis and review of existing literature. Revista de Biología Tropical, Vol. 62 (Suppl. 2): 153-165.

Reyes-Morales, F., \& Springer, M. (2014). Efecto del esfuerzo de muestreo en la riqueza de taxa de macroinvertebrados acuáticos y el índice BMWP/ Atitlán. Revista de Biología Tropical, Vol. 62 (Supl. 2): 289-299.

Rico-Sánchez, A. E., Rodríguez-Romero, A. J., LópezLópez, E., \& Sedeño-Díaz, J. E. (2014). Patrones de variación espacial y temporal de los macroinvertebrados acuáticos en la Laguna de Tecocomulco, Hidalgo (México). Revista de Biología Tropical, Vol. 62 (Supl. 2): 79-94.

Rios-Touma, B., Acosta, R., \& Prat, N. (2014). The Andean Biotic Index (ABI): revised tolerance to pollution values for macroinvertebrate families and index performance evaluation. Revista de Biología Tropical, Vol. 62 (Suppl. 2): 247-271.

Rincón, J., \& Covich, A. (2014). Effects of insect and decapod exclusion and leaf litter species identity on breakdown rates in a tropical headwater stream. Revista de Biología Tropical, Vol. 62 (Suppl. 2): 141-152.

Stanford, J. A., \& Covich, A. P. 1988. Preface. Journal of the North American Benthological Society 7: 261-262.

Torres-García, U., Pérez-Valladares, C. X., Herrería-Diego, Y., \& Pineda-López, R. F. (2014). Efecto de los factores ambientales sobre la diversidad de insectos hemimetábolos y coleópteros acuáticos en la cuenca del Río Xichú, Guanajuato, México. Revista de Biología Tropical, Vol. 62 (Supl. 2): 67-78. 
Torres, P. J., \& Ramírez, A. (2014). Land use effects on leaf litter breakdown in low-order streams draining a rapidly developing tropical watershed in Puerto Rico. Revista de Biología Tropical, Vol. 62 (Suppl. 2): $127-140$.

Vásquez-Ramos, J. M., Guevara-Cardona, G., \& ReinosoFlórez, G. (2014). Factores ambientales asociados con la preferencia de hábitat de larvas de tricópteros en cuencas con bosque seco tropical (Tolima,
Colombia). Revista de Biología Tropical, Vol. 62 (Supl. 2): 19-38.

Vinson, M. R., \& Hawkins, C. P. (2003). Broad-scale geographical patterns in local stream insect genera richness. Ecography, 26: 751-767.

Wantzen, K. M., Ramirez, A. \& Winemiller, K. O. (2006). New vistas in Neotropical stream ecology-preface. Journal of the North American Benthological Society, 25: 61-65. 